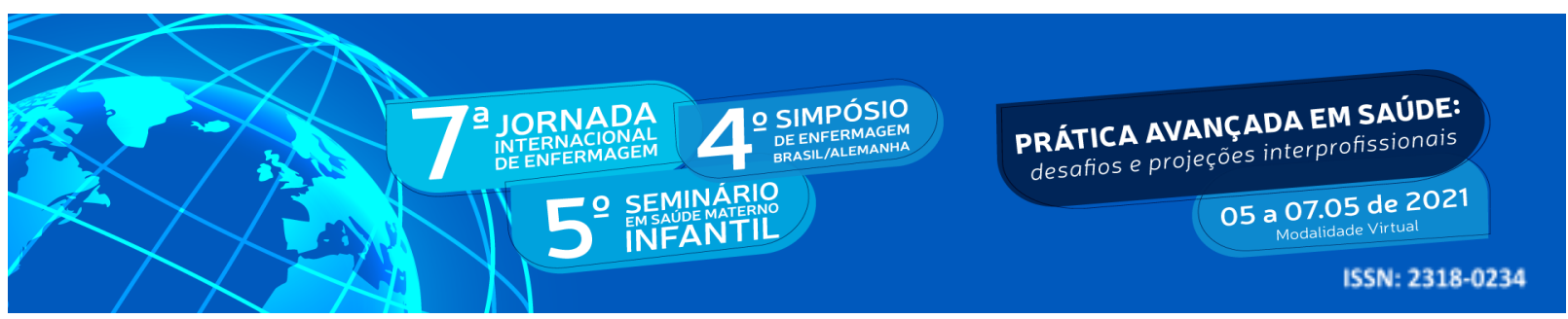

DOI: http://doi.org/10.48195/jie2021-135

\title{
O CONSUMIDOR QUER SABER MAIS SOBRE OS ALIMENTOS EM SERVIÇOS DE ALIMENTAÇÃO' ${ }^{1}$
}

\author{
Natalia Brugalli ${ }^{2}$; Patricia Arruda Scheffer ${ }^{3}$; Cátia Regina Storck ${ }^{4}$; Ana Lúcia de \\ Freitas Saccol ${ }^{5}$
}

\begin{abstract}
RESUMO
Este estudo teve como objetivo avaliar a satisfação e a influência nas escolhas dos consumidores quanto à presença de informativos nutricionais em serviços de alimentação. Realizou-se em março de 2021 por meio de formulário no Google Forms ${ }^{\circledR}$ com 14 questões, divulgou-se nas redes sociais por 10 dias, para consumidores do Rio Grande do Sul maiores de 19 anos. Obteve-se 87 respostas, 79,3\% foram mulheres, e a média de idade da amostra resultou em 38,03. Relataram frequentar os restaurantes e lanchonetes antes da Pandemia de Covid-19, e no período atual pedem refeições para consumo em casa. O estudo mostrou que os consumidores gaúchos não estão satisfeitos com as informações presentes nos alimentos expostos nos serviços de alimentação, gostariam de ter acesso a lista de ingredientes e alertas sobre o excesso de sódio e açúcar. Acredita-se que a presença de informativos nutricionais possa contribuir com escolhas mais conscientes e na promoção de hábitos alimentares mais saudáveis.
\end{abstract}

Palavras-chave: Hábitos Alimentares; Informativos Nutricionais; Pandemia; Saúde.

\begin{abstract}
This study aimed to evaluate satisfaction and influence on consumer choices regarding the presence of nutritional information in food services. It took place in march 2021 through a form on Google Forms ${ }^{\circledR}$ with 14 questions, it was published on social networks per 10 days, for consumers in Rio Grande do Sul over 19 years old. 87 responses were obtained, 79,3\% were women. and the mean age of the sample resulted in 38,03125. They reported attending restaurants and cafeterias before the Covid-19 pandemic, and in the current period they order meals for consumption at home. The study showed that consumers in Rio Grande do Sul are not satisfied with the information present in the food exposed in food services, they would like to have access to the list of ingredients and alerts about sodium and sugar excess. It is believed that the presence of nutritional information can contribute to more conscious choices and the promotion of healthier eating habits.
\end{abstract}

Key Words: Eating Habits; Health; Nutritional Information; Pandemic.

\footnotetext{
${ }^{1}$ Projeto de Iniciação Tecnológica. Bolsa PIBITI/CNPq. Universidade Franciscana - UFN

${ }^{2}$ Estudante do Curso de Nutrição. Universidade Franciscana - UFN. E-mail: nati.brugalli@ufn.edu.br

${ }^{3}$ Nutricionista. Mestranda em Ciências da Saúde e da Vida- Universidade Franciscana.- UFN. Grupo de Pesquisa em Segurança Alimentar e Nutricional (GESAN/CNPq). E-mail: patricia.scheffer@ufn.edu.br

${ }^{4}$ Coorientador. Professora Doutora Curso de Nutrição. Universidade Franciscana. Grupo de Pesquisa em Segurança Alimentar e Nutricional (GESAN/CNPq). E-mail: çatiars@ufn.edu.br

${ }^{5}$ Orientadora Professora Doutora Curso de Nutrição e Mestrado em Ciências da Saúde e da Vida. Universidade Franciscana - UFN. Grupo de Pesquisa em Segurança Alimentar e Nutricional (GESAN/CNPq). E-mail: alsaccol@ufn.edu.br
} 


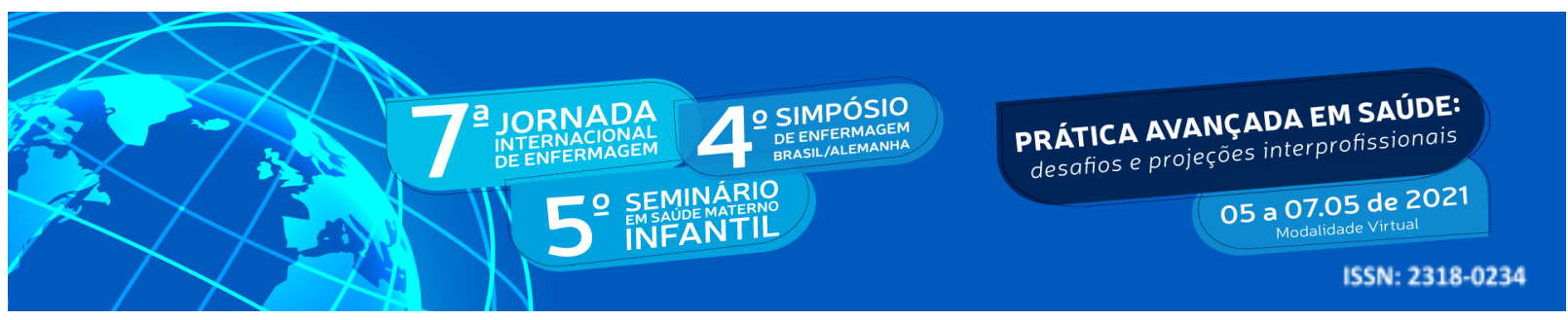

\section{INTRODUÇÃO}

Os consumidores no mundo inteiro procuram, cada vez mais, informações sobre os alimentos que consomem. Uma fonte importante para obter esse tipo de informação são os rótulos dos alimentos, que, muitas vezes, dispõem de dados sobre o conteúdo nutricional, bem como frases que relacionam o seu consumo com benefícios para a saúde. A disponibilização de informações adequadas e compreensíveis sobre o conteúdo nutricional, que não levem o consumidor a erro, pode contribuir para a promoção da saúde e a redução do risco de doenças relacionadas à alimentação e à nutrição (COUTINHO; RECINE, 2007).

A questão de acordo com Grunert e colaboradores (2010) é sobre como os informativos nutricionais devem ser criados para ajudar quem consome a realizar escolhas conscientes e benéficas à saúde. Existem fatores que influenciam a atenção do consumidor a um determinado produto, como por exemplo, o design da embalagem, as informações presentes e como elas estão dispostas, por este motivo é necessário pensar qual a melhor maneira de alertar nutricionalmente o consumidor, pois informando da forma correta pode-se estimular boas escolhas alimentares e por consequência bons hábitos alimentares, desta forma, reduzindo as chances de adquirir doenças como obesidade, diabetes, hipertensão, problemas no coração, entre outras (GRUNERT et al., 2010).

A Política Nacional de Promoção da Saúde (BRASIL, 2010) enfatiza a necessidade de estimular ações de empoderamento da informação pelo consumidor, de modo a alcançar o entendimento e uso prático da rotulagem geral e nutricional dos alimentos. Como o consumo alimentar é uma das determinantes positivas ou negativas da saúde, as intervenções de cunho educativo destacam-se, constituindo uma estratégia fundamental das políticas de saúde de abrangência global, despontando como uma ferramenta para a redução da obesidade e das Doenças Crônicas Não Transmissíveis (DCNT) (SANTOS et al., 2016).

Em relação à disponibilização de algum tipo de informação nutricional em restaurantes, a partir de 2001, começaram a surgir legislações relacionadas à temática, mas ainda restritas a alguns municípios e estados brasileiros, como Distrito Federal, Rio de Janeiro (RJ), Sorocaba (SP) e ao Estado de Santa Catarina. Apesar da abrangência limitada dessas regulamentações, 


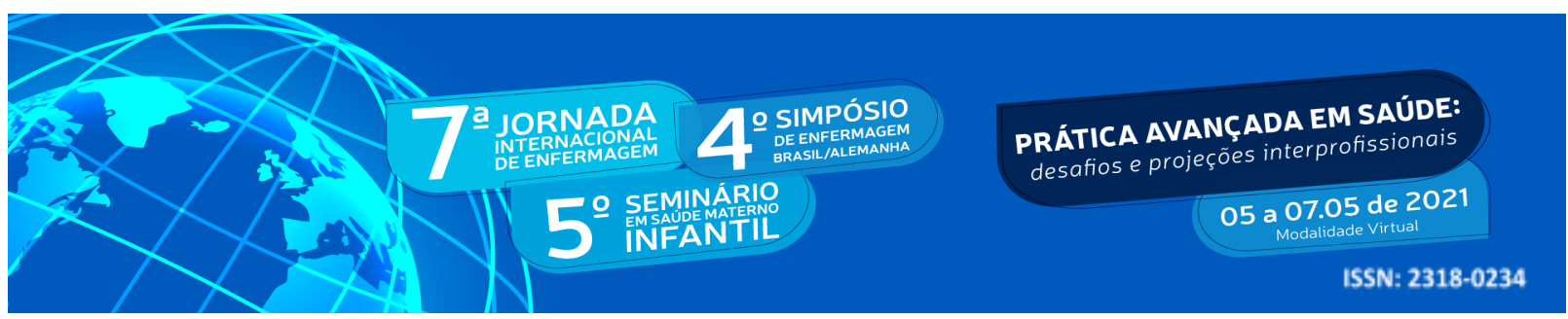

seja em relação ao território ou ao conteúdo, elas podem ser reflexo da preocupação da população em relação à sua saúde e ao direito de informação sobre os alimentos consumidos fora de casa (SANCHES et al., 2013).

Portanto, a existência de informações nutricionais em restaurantes é importante no sentido de oferecer ao consumidor dados sobre a composição dos alimentos oferecidos nos restaurantes. Porém, o consumidor pode considerar a presença dessas informações como fator determinante no processo de escolha do restaurante em que vai se alimentar, como também pode considerá-las importantes ou não e utilizá-las ou não (LIMA-FILHO et al., 2014).

\section{OBJETIVO}

Este estudo teve como objetivo avaliar a satisfação e a influência nas escolhas dos consumidores quanto à presença de informativos nutricionais em serviços de alimentação.

\section{METODOLOGIA}

Pensando na situação atual de pandemia devido a Covid-19, optou-se por elaborar um questionário on-line no Google Forms ${ }^{\circledR}$ para consumidores com idade a partir dos 19 anos e que residem em Porto Alegre, Santa Maria ou Santana do Livramento, Rio Grande do Sul, Brasil. A pesquisa então foi encaminhada através das mídias sociais entre os dias 5 e 15 de março de 2021. Este estudo compõe resultados parciais de uma pesquisa maior, onde futuramente serão realizadas comparações estatísticas entre os municípios, considerando as diferenças de números de habitantes.

O formulário enviado foi composto por catorze questões ao todo, sendo a parte inicial um Termo de Consentimento Livre e Esclarecido (TCLE), onde há informações como o intuito da pesquisa, o responsável, a duração; também se esclarece que não é necessário informar a identidade e não há custos, os contatos que podem ser úteis para eventuais dúvidas e mais alguns detalhes para que o respondente fique ciente do que realizará.

$\mathrm{Na}$ primeira parte do formulário, buscou-se saber mais sobre características pessoais básicas e o município que residia, caso não fizesse parte de uma das três cidades selecionadas, o formulário era descartado. Indagou-se sobre a frequência que os participantes vão aos 


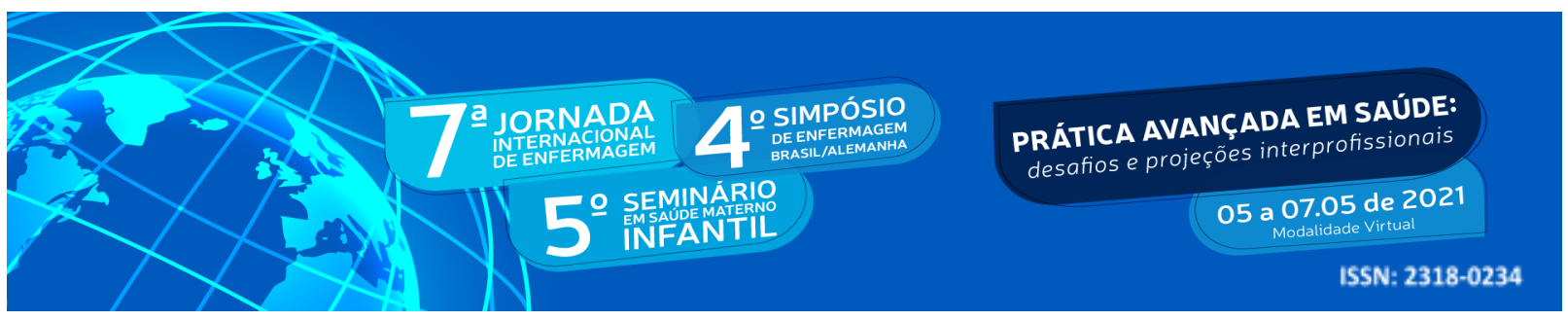

serviços de alimentação considerando a rotina antes da pandemia e a frequência com que pede refeições e/ou lanches em sua residência considerando a pandemia, sempre utilizando a escala de Likert (LIKERT, 1932).

$\mathrm{Na}$ segunda parte, aprofunda-se mais no assunto quanto a presença dos informativos nutricionais em serviços de alimentação, qual a satisfação dos respondentes quanto ao que há disponível atualmente e quais informações nutricionais podem ser úteis de visualizar nos alimentos e preparações além da identificação e preço. Questionou-se também sobre as mudanças nas escolhas alimentares caso estivessem disponíveis mais informações acerca dos alimentos que consomem e se acreditam que os serviços de alimentação precisam oferecer mais detalhes nutricionais dos seus produtos. Após finalizar o questionário havia uma questão extra objetiva e sem obrigatoriedade de resposta, onde indagava-se qual a melhor forma de implementar e utilizar as informações nutricionais nos alimentos, podendo também escolher a alternativa "não sei opinar.

Os dados foram analisados por meio de estatística descritiva simples, tabulados em planilhas do Microsoft Excel e apresentados no formato de gráficos e tabelas, com média e percentuais. O presente estudo foi aprovado no Comitê de Ética da Universidade Franciscana sob o número CAAE 62711216.8.0000.5306 e parecer número 1.877.129. Faz parte de um Projeto maior intitulado "Elaboração de software para desenvolvimento de informativos nutricionais para serviços de alimentação".

\section{RESULTADOS E DISCUSSÃO}

Com a aplicação do formulário on-line obteve-se 92 respondentes, 5 foram excluídos pois não residiam nos municípios foco da pesquisa, restando 87 consumidores na amostra. Os municípios selecionados possuem três classificações diferentes de acordo com o último censo do Instituto Brasileiro de Geografia e Estatística (IBGE), Santana do Livramento sendo um centro sub-regional B, Santa Maria uma capital regional C e Porto Alegre uma metrópole C, (IBGE, 2010), desta forma conseguiu-se avaliar distintas regiões com tamanho e quantidade de habitantes variados 


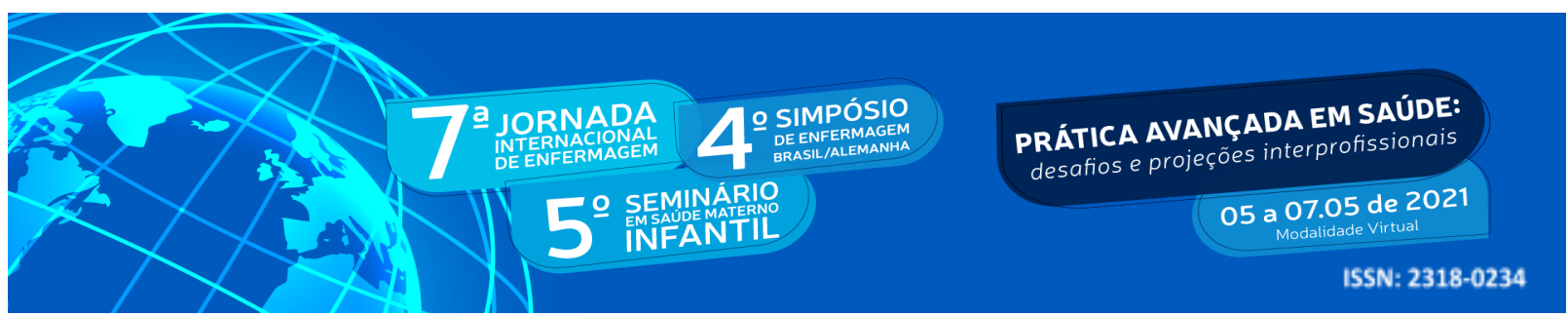

$\mathrm{Na}$ amostra de 87 pessoas, a grande maioria era do sexo feminino (79,3\%). Deste total, 55,2\% residem em Santa Maria, 27,6\% em Santana do Livramento e 17,2\% de Porto Alegre. Com a continuidade do estudo pretende-se obter uma amostra mais homogênea entre os municípios, para possibilitar comparações mais exatas. A média entre as idades é de 38,03 e o desvio padrão igual a 12,86.

Em relação à frequência com que os respondentes vão aos restaurantes comerciais considerando a rotina antes da pandemia, nota-se que a maioria vai a estes locais frequentemente $(29,9 \%)$ ou muito frequentemente (19,5\%), ninguém referiu nunca frequentar. Quanto aos bares e lanchonetes, a maioria respondeu frequentar ocasionalmente antes da pandemia $(34,39,1 \%)$. Pode-se verificar uma frequência maior de utilização dos serviços de alimentação do tipo restaurantes quando comparados às lanchonetes e bares. Bem como mais da metade dos consumidores relataram pedir alimentos por meio de delivery, durante o período pandêmico. Na figura 1 pode-se observar os resultados destas questões.

Figura 1: Resultados referente a frequência dos consumidores em restaurantes comerciais, lanchonetes e/ou bares e pedidos de refeições em suas residências, Rio Grande do Sul, Brasil, 2021.

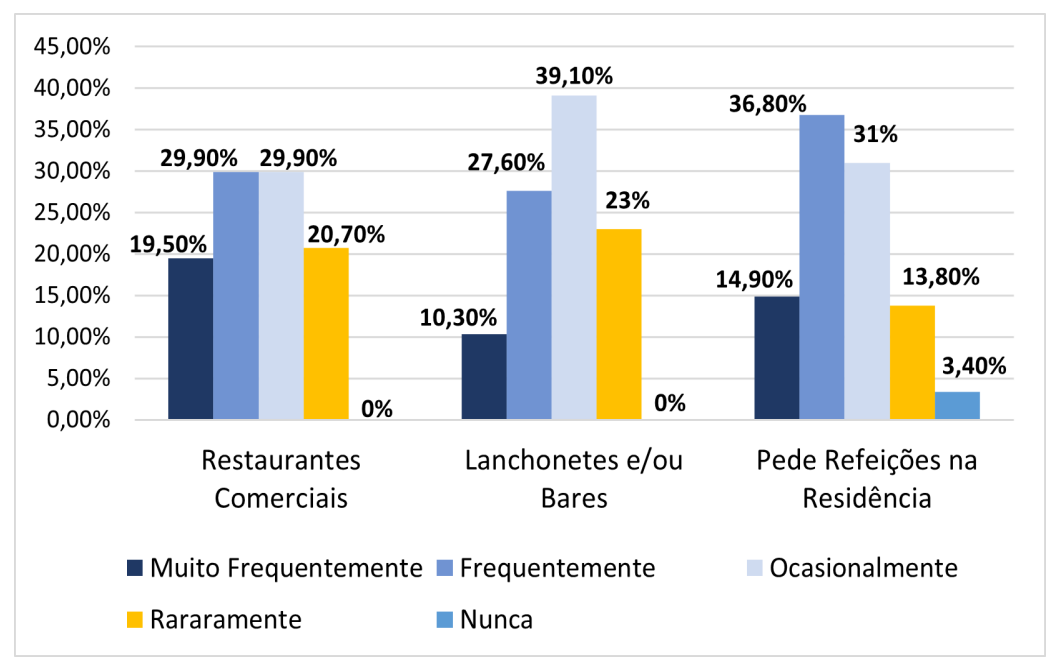

A alimentação fora de casa ascendeu muito nas últimas décadas, um estudo comparou o tempo que as mulheres dedicavam-se para preparar as refeições para sua família em 1965 e em 2008, os autores concluíram que cada vez mais as mulheres dedicam menos tempo para cozinhar em casa e desta forma acabam realizando as refeições fora do lar (SMITH et al., 


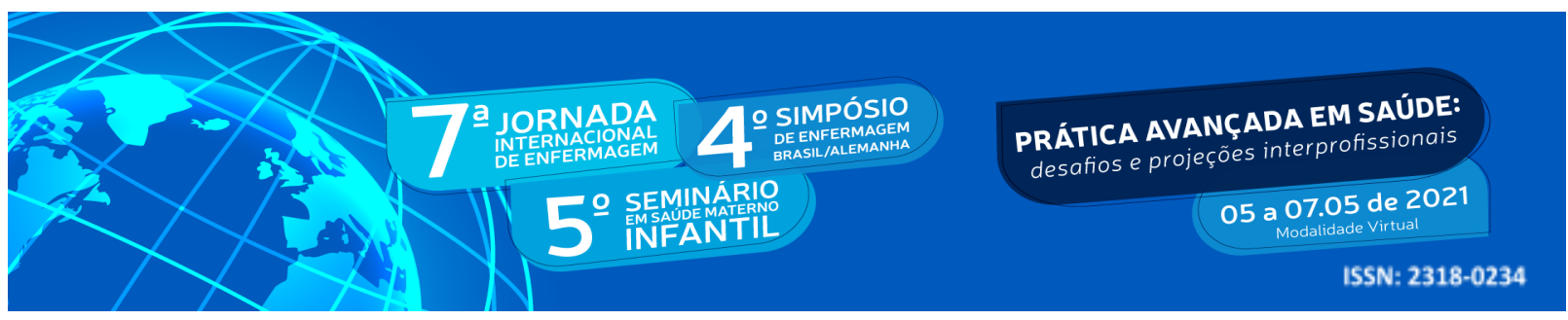

2013). Isto deve-se ao fato que o público feminino está cada vez mais inserido no mercado de trabalho, diferente de como acontecia no século passado (QUEIROZ; COELHO, 2017).

Percebe-se uma quantidade majoritária de pessoas insatisfeitas com as informações disponíveis em preparações e alimentos de buffets e balcões expositores de restaurantes, lanchonetes e bares, mais de $70 \%$ dos participantes gostariam de saber mais do que apenas o preço e o nome do produto. Este resultado é interessante, pois mostra que a presença de informativos nutricionais em serviços de alimentação pode ser uma tendência no mercado de alimentação coletiva. Alguns autores internacionais já estão pesquisando a presença dos informativos nutricionais em serviços de alimentação, demonstrando sua importância para a população (CHRISTOPH; ELLISON, 2017; GOMEZ; LE MINOUS, 2012).

Este modelo de rotulagem em cardápio poderá vir a ser um diferencial para este setor, de acordo com Fitzgerald (2018) as empresas utilizam da rotulagem em serviços de alimentação como uma estratégia de marketing. Na tabela 1 observa-se o posicionamento dos pesquisados no que tange ao item de satisfação com as informações disponíveis em serviços de alimentação específicos.

Tabela 1: Satisfação de consumidores quanto às informações disponíveis em preparações e alimentos de buffets e balcões expositores, Rio Grande do Sul, Brasil, 2021.

\begin{tabular}{l|c|c}
\hline $\begin{array}{c}\text { Satisfação ao ver apenas preço e } \\
\text { nome do produto nas preparações em } \\
\text { restaurantes e lanchonetes }\end{array}$ & Número de pesquisados & Porcentagem \\
\hline SIM (satisfeito) & 16 & $18,4 \%$ \\
NÃO (insatisfeito) & 63 & $72,4 \%$ \\
NÃO SEI OPINAR & 8 & $9,2 \%$ \\
\hline Total & 87 & $100 \%$ \\
\hline
\end{tabular}

De forma objetiva perguntou-se quais informações além do preço e identificação os pesquisados gostariam de visualizar nas refeições e lanches, sendo possível selecionar mais de uma alternativa, entre elas lista de ingredientes, calorias, tamanho da porção, porcentagem de macronutrientes, benefícios para a saúde, alto teor/fonte de fibras, alertas de excesso de sódio, gordura e açúcar (sendo esta opção separada em 3 alternativas diferentes), presença de alergênicos, nenhuma informação e outras informações que puderam ser citadas na questão 


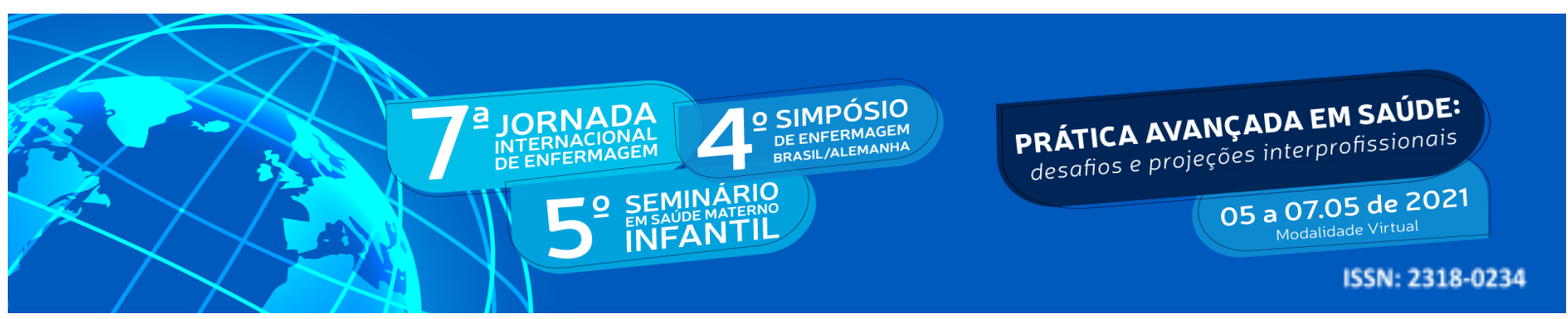

seguinte. Apenas um respondente selecionou esta opção com resposta aberta e sugeriu que seria interessante adicionar a informação de produto vegano ou vegetariano. Lista de ingredientes, alerta de excesso de sódio e de açúcar foram as informações mais solicitadas, em torno de $70 \%$ dos participantes. Na figura 2 encontram-se os resultados do questionamento sobre informações adicionais nos alimentos e preparações, além de sua identificação e preço em formato de gráfico.

Figura 2: Porcentagem por alternativa de quais informações nutricionais adicionais seriam interessantes de visualizar nos alimentos e preparações, Rio Grande do Sul, Brasil, 2021.

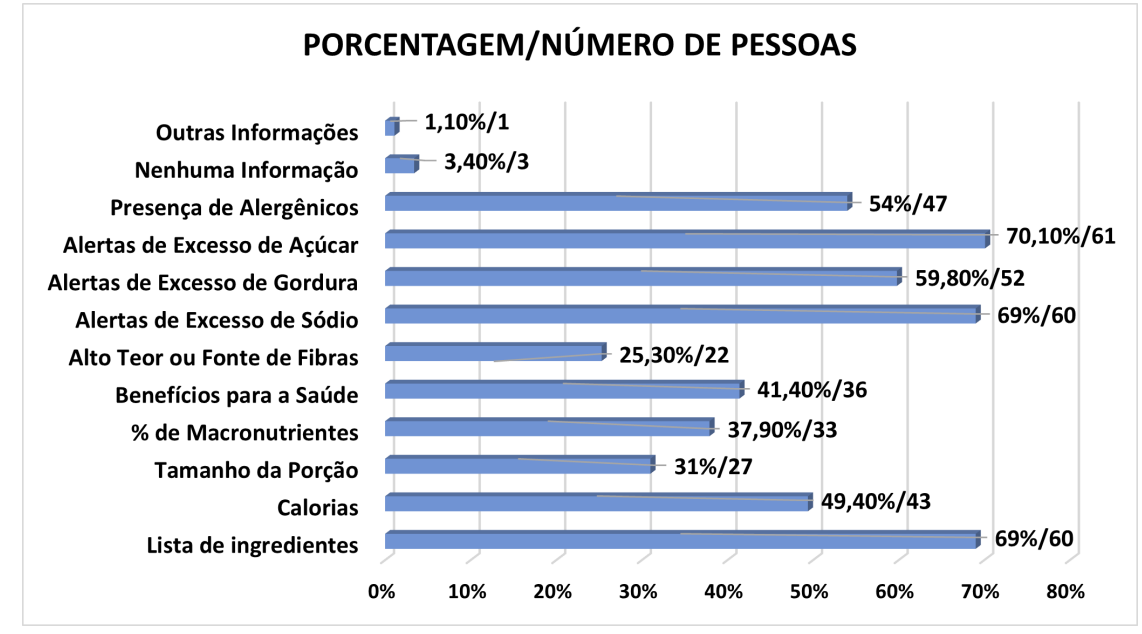

Estudos recentes demonstraram que a rotulagem em serviços de alimentação é muito importante para os frequentadores destes locais (CHRISTOPH; ELLISON, 2017). No nosso estudo, percebe-se a importância dos informativos nutricionais com relação a mudança de hábitos, na questão que se refere ao quanto esses dados impactam nas escolhas por parte do consumidor.

A maioria $(64,4 \%)$ trocaria de opção caso houvesse mais informações acerca das preparações disponíveis em restaurantes, lanchonetes e bares. A longo prazo pode-se ter uma melhora do estilo e qualidade de vida da população.

Quanto ao porquê trocariam suas escolhas alimentares encontram-se as mais diversas respostas como "Pela saúde", "Para saber ao certo o que se está consumindo", "Porque tenho alergias alimentares", "Saberia o que faz bem para minha saúde", "Pelos benefícios", "Para não engordar", "Para ter noção dos ingredientes que estou ingerindo", "Para escolher a opção 


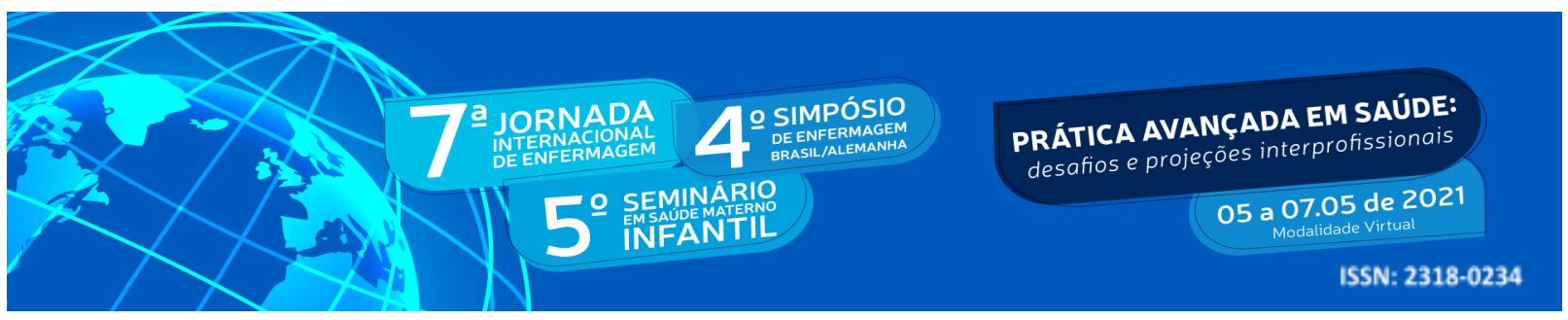

mais saudável", "Para ficar mais segura", "Para não consumir tanto açúcar e sal", "Para melhorar os hábitos", dentre outros motivos. Esta foi uma questão com resposta aberta que no projeto maior será realizada a análise de conteúdo.

Para White e colaboradores (2016), a implementação deste tipo de rotulagem em cardápios ainda não é investida pelos restaurantes, do ponto de vista dos autores isto ocorre devido ao fato que este tipo de informação não é obrigatória, desta maneira acaba que os proprietários não aderem a tal estratégia (WHITE et al., 2016).

Já em nossos resultados, os respondentes no geral, acreditam quase que de maneira unânime, $84(96,6 \%)$ dentre os 87 pesquisados que há a necessidade dos restaurantes, lanchonetes e bares disponibilizarem mais informações acerca de seus produtos. Apenas 3 pessoas relataram que não, e não houve ninguém que não soube opinar.

$\mathrm{Na}$ questão extra, ao final do formulário, a qual trata sobre a implementação e a melhor forma de utilizar e comparar informativos nas preparações e alimentos dispostos em restaurantes e lanchonetes. Observou-se que a maior parcela de respondentes (51) escolheu que gostaria de analisar as informações na porção de consumo, de forma que não seria possível comparar dois produtos distintos. Outra parte da amostra (25) prefere comparar pela quantidade, como por exemplo os nutrientes entre $100 \mathrm{~g}$ de um alimento e $100 \mathrm{ml}$ de outro. Não souberam responder apenas 11 pessoas. Pode-se pensar em uma estratégia de se apresentar as informações dos alimentos das duas formas, tanto na porção quanto em $100 \mathrm{~g} / \mathrm{ml}$. A figura 3 mostra o gráfico com resultados percentuais da questão final do formulário, onde todos os participantes responderam mesmo sem obrigatoriedade.

Figura 3: Porcentagens por alternativa, referente a opinião dos pesquisados sobre a implementação e utilização dos informativos nutricionais nas preparações, Rio Grande do Sul, Brasil, 2021.

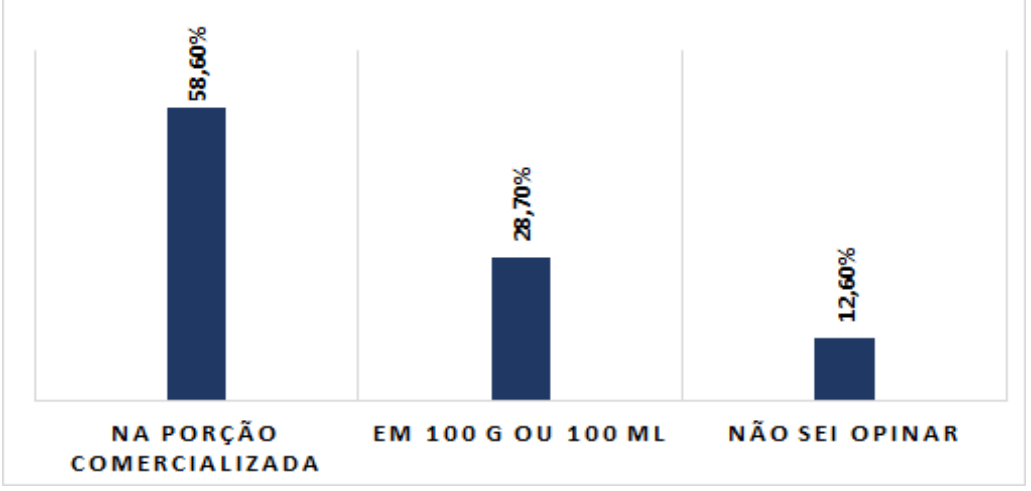




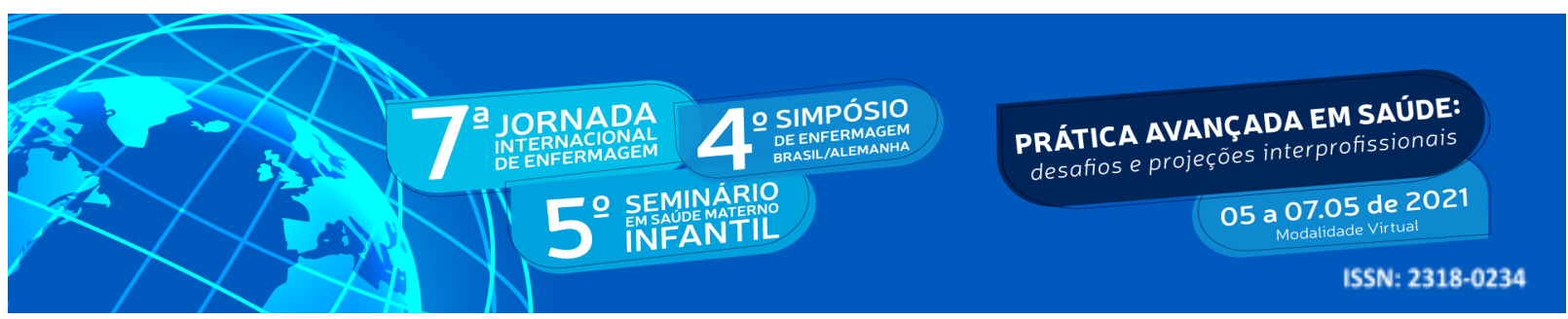

\section{CONCLUSÃO}

Com os resultados parciais desta pesquisa pode-se dizer que a maioria frequentava os restaurantes e lanchonetes antes da Pandemia do Covid-19, e no período atual pede refeições para consumo em casa. O estudo mostrou que os consumidores gaúchos não estão satisfeitos com as informações presentes nos alimentos expostos nos serviços de alimentação, gostariam de ter acesso a lista de ingredientes e alertas sobre o excesso de sódio e açúcar.

Acredita-se que a presença de informativos nutricionais possa contribuir com escolhas mais conscientes e na promoção de hábitos alimentares mais saudáveis. Mostra-se necessário aprofundar a pesquisa e aprimorar o formulário de questões para adentrar cada vez mais neste assunto e complementar o que foi estudado até o momento.

\section{REFERÊNCIAS}

BRASIL. Política Nacional de Promoção da Saúde (PNPS). BRASÍLIA: Ministério da Saúde, 2010. [Citado em 19 Março 2021]. Disponível em:

https://bvsms.saude.gov.br/bvs/publicacoes/politica nacional promocao saude 3ed.pdf

COUTINHO, J. G.; RECINE, E. Experiências Internacionais de Regulamentação das Alegações de Saúde em Rótulos de Alimentos. Brasília: Revista Panamericana de Salud Publica, 2007.

CHRISTOPH, M. J.; ELLISON, B. A Cross-Sectional Study of the Relationship between Nutrition Label Use and Food Selection, Servings, and Consumption in a University Dining Setting. Journal of the Academy of Nutrition and Dietetics, v. 117, n. 10, p. 1528-1537, out. 2017.

FITZGERALD, S. ET AL. An evaluation and exploration of Irish food-service businesses' uptake of and attitudes towards a voluntary government-led menu energy (calorie) labelling initiative. Public Health Nutrition, 2018.

GOMEZ, P.; LE MINOUS, A. E. L'influence du format de l'étiquetage sur l'utilisation et la compréhension de l'information nutritionnelle: Résultats d'une expérimentation menée en restauration collective. Revue d'Epidemiologie et de Sante Publique, v. 60, n. 1, p. 9-18, 2012.

GRUNERT, K.G. ET AL. Use and Understanding of Nutrition Information on Food Labels in Six Europeans Countries. Aarhus: Journal of Public Health, 2010.

INSTITUTO BRASILEIRO DE GEOGRAFIA E ESTATÍSTICA (IBGE). Censo Demográfico; 2010. [Citado em 11 Março 2021]. Disponível em: https://cidades.ibge.gov.br/. 


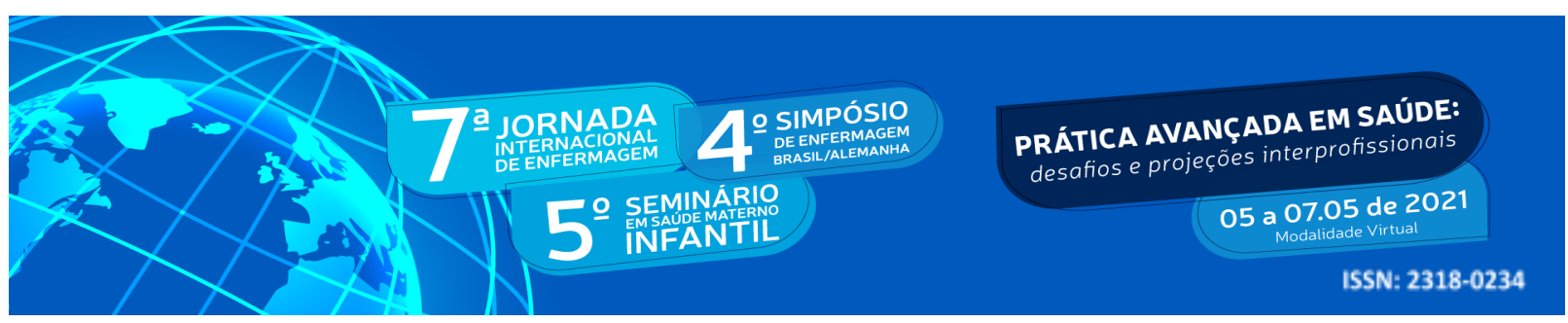

LIKERT, R. A Technique for the Measurement of Attitudes. New York: Archives in Psychology, 1932.

LIMA-FILHO, D. O. ET AL. Impacto das Informações Nutricionais no Comportamento do Consumidor de Restaurantes. Minas Gerais: Organizações Rurais \& Agroindústria, 2014.

QUEIROZ, P. W. V. DE; COELHO, A. B. Alimentação Fora De Casa: Uma Investigação Sobre Os Determinantes Da Decisão De Consumo Dos Domicílios Brasileiros. Análise Econômica, v. 35, n. 67, 2017.

SANTOS, C. M. B. ET AL. Experiência de Extensão: "Rotulagem Nutricional: conheça o que você consome”. São Paulo: Revista Ciência em Extensão, 2016.

SANCHES, M. Z. ET AL. Disponibilidade de Informação Nutricional em Restaurantes no Município de São Paulo. São Paulo: DEMETRA: Alimentação, Nutrição \& Saúde, 2013.

SILVA, T.B.C. ET AL. Modelos de Rotulagem Nutricional Frontal de Alto Conteúdo de Nutrientes Críticos Comparado a Outros Modelos de Rotulagem Frontal Nutricional:

Uma Revisão Sistemática. Brasília: 2019.

SMITH, L. P. ET AL. Trends in US home food preparation and consumption: Analysis of national nutrition surveys and time use studies from 1965-1966 to 2007-2008. Nutrition Journal, v. 12, n. 1, p. 1-10, 2013.

WHITE, C. M. ET AL. A voluntary nutrition labeling program in restaurants: Consumer awareness, use of nutrition information, and food selection. Preventive Medicine Reports, v. 4, p. 474-480, 1 dez. 2016 